\title{
Digital Data Processing Based on Wavelet Transforms
}

\author{
Olga Timoshevskaya \\ Institute of Engineering Sciences \\ Pskov State University \\ Pskov, Russia \\ olga.tim777@yandex.ru
}

\author{
Victor Samsonenkov \\ Institute of Engineering Sciences \\ Pskov State University \\ Pskov, Russia \\ viktor_psk@yahoo.com
}

\author{
Vladimir Londikov \\ Institute of Engineering Sciences \\ Pskov State University \\ Pskov, Russia \\ redcat60@mail.ru \\ Tatyana Klets \\ Institute of Humanities and Linguistic \\ Communications \\ Pskov State University \\ Pskov, Russia \\ kte63@yandex.ru
}

\author{
Dmitry Andreev \\ Institute of Engineering Sciences \\ Pskov State University \\ Pskov, Russia \\ dandreev60@mail.ru
}

\begin{abstract}
The paper focuses on the main theoretical principles and properties of wavelet transforms. The problem of digital data processing based on wavelet transforms is considered. The analysis and processing of signals and functions that are non-stationary in time and inhomogeneous in space are presented. The authors propose methods of progressive coefficients' values that combine wavelet decomposition and quantization, the main purpose of which is to convey the most important piece of information about a signal.
\end{abstract}

Keywords - basic wavelet function, discrete (DWT) and continuous (CWT) wavelet transform, scaling function, wavelet transform.

\section{INTRODUCTION}

Currently, information systems and technologies are actively used in almost all spheres of human life. In this regard, the constant growth of the volume of digital information is characteristic, the increasing requirements for the quality of its processing, transmission and storage $[1,2]$. The results of research in the field of digital information processing have found application: in mechanical engineering and instrument making, in the military field, in medicine and ecology, in geographic information systems and cartography, in scientific research and other areas.
As a rule, various compression methods are often used to reduce the amount of information during its storage and processing. At the same time, it is necessary that the compressed data contain only the most significant and unique part of it, according to which it is possible to restore all the original information.

There are a large number of data compression methods that are used to achieve different purposes and, as a result, have their own characteristics. Relatively new, but at the same time popular due to the extensive field of application of this mathematical apparatus, is the scientific direction associated with the theory of wavelets.

\section{DEFINING A WAVELET TRANSFORM}

Wavelet is a generalized name for a family of mathematical functions local in time and in frequency, which have the form of a small wave with nonzero values in the central region and decrease to zero with distance from the center. Wavelets include functions that are constructed from one parent wavelet $\psi(t)$ by time shift operations (b) and time scale changes (a): 


$$
\begin{aligned}
& \psi_{a b}(t)=\frac{1}{\sqrt{|a|}} \cdot \psi\left(\frac{t-b}{a}\right), \\
& (a, b) \in R, \psi(t) \in L^{2}(R)
\end{aligned}
$$

Wavelet transform is a transform that transforms a signal from a temporal representation into a timefrequency representation and is a convolution of a wavelet function with a signal [3]. The results of the wavelet transform contain combined information about the analyzed signal and the wavelet itself, and also allow you to isolate the low-frequency and high-frequency components of the signal.

In digital data processing, it is necessary to comply with some requirements related to scaling and orientation of objects, as well as spatial localization. Data transformation should allow analyzing the image of an object simultaneously at different scales. Spatial localization of transformation is usually resorted to when information about the location of object details is necessary.

\section{DISCRETE (DWT) AND CONTINUOUS (CWT) WAVELET TRANSFORMS}

For signal processing and transformation, as a rule, discrete (DWT) and continuous (CWT) wavelet transforms are used.

Let the functions $s(t)$ with finite energy (norm) in space $L^{2}(R)$, be defined along the whole real axis $R(-\infty, \infty)$.

Continuous wavelet transform or wavelet image function $s(t) \in L^{2}(R)$ is a function of two variables:

$$
\begin{aligned}
& C(a, b)=\left(\frac{1}{\sqrt{|a|}}\right) \int_{-\infty}^{\infty} s(t) \cdot \psi\left(\frac{t-b}{a}\right) d t, \\
& (a, b) \in R, a \neq 0
\end{aligned}
$$

where $s(t)$ - original function, $\psi(t)$ - mother wavelet function, $a$ - wavelet timescale parameter, $b-$ wavelet time offset.

Analysis by continuous wavelet transform starts at high frequencies and is carried out in the direction of their reduction. The first value of the scaling parameter corresponds to the most compressed wavelet, then the value is increased and the wavelet is expanded. The wavelet is placed at the beginning of the signal, multiplied with it, integrated and normalized. The result of the calculation is placed at the point of the time scale of the transformation spectrum corresponding to the unit time parameter and the zero time offset of the wavelet. At the next step, the unit scale wavelet is shifted to the right by the value $b$ and the calculations are repeated. The procedure is repeated until the wavelet reaches the end of the signal. The result is a row of points on a time scale for a unit scale. To calculate the next scale row, the time scale value is increased by some value.

Thus, the continuous wavelet transform is the decomposition of the signal in terms of all possible shifts and contractions / stretches of some localized finite function - a wavelet. In this case, the variable ' $a$ ' determines the wavelet scale and is equivalent to the frequency in Fourier transforms, and the variable ' $b$ ' is the wavelet shift from the initial point in the domain of its definition, the scale of which completely repeats the time scale of the analyzed signal. Hence it follows that wavelet analysis is a frequency-spatial analysis of signals.

Continuous wavelet transform is poorly suited for numerical calculations. Also, this transformation gives wavelet spectra with an excessive amount of information, that is, it is possible to completely restore the signal with a smaller number of spectral components. Continuous wavelet transform uses the entire range of variation of the values of $a$ and $b$, which in practice is impossible to achieve. A choice of samples with respect to $a$ and $b$ is required, or sampling of the phase space [4], which would eliminate this redundancy.

Therefore, in practice, discrete wavelet transform is most often used.

Discrete wavelet transform provides enough information for both signal analysis and synthesis, while being economical in terms of the number of operations and the required memory. DWT operates with discrete parameter values $a$ and $b$, which are set, as a rule, in the form of power functions:

$$
\begin{aligned}
& a=a_{0}^{-m}, b=k \cdot a_{0}^{-m}, \\
& a_{0}>1, m, k \in Z
\end{aligned}
$$

where $m$ - scale parameter, $k$ - shift parameter [5].

The direct transform wavelet coefficients are described by the following relation:

$$
C_{m k}=\left|a_{0}\right|^{\frac{m}{2}} \int_{-\infty}^{\infty} s(t) \cdot \psi\left(a_{0}^{m} t-k\right) d t
$$

The inverse discrete transformation for continuous signals with a normalized orthogonal wavelet basis of space is represented in the following form:

$$
s(t)=\sum_{m=-\infty}^{\infty} \sum_{k=-\infty}^{\infty} C_{m k} \cdot\left|a_{0}\right|^{\frac{m}{2}} \cdot \psi\left(a_{0}^{m} t-k\right)
$$

When processing data arrays, discrete wavelets are used in a pair with their associated discrete scaling or scaling functions $\varphi_{m k}(t)$. If wavelets are considered as analogs of high-frequency filters of a signal, then the scaling functions are analogs of low-frequency filters, with which the components that have not passed the wavelet filtering are extracted from the signal. The sum of the wavelet coefficients and the scaling coefficients of the signals' decomposition provides the ability to perform accurate signals' reconstruction: 
Environment. Technology. Resources. Rezekne, Latvia Proceedings of the $13^{\text {th }}$ International Scientific and Practical Conference. Volume 2, 174-180

$$
\begin{aligned}
& s(t)=\sum_{k=-\infty}^{\infty} C_{a_{k}} \cdot \varphi_{k}(t)+ \\
& +\sum_{m=-\infty}^{\infty} \sum_{k=-\infty}^{\infty} C_{d_{m k}} \cdot\left|a_{0}\right|^{\frac{m}{2}} \cdot \psi\left(a_{0}^{m} t-k\right)
\end{aligned}
$$

where $C_{a_{k}}-$ scaling factors, or signal approximation factors, $C_{d_{m k}}$ - wavelet coefficients, or detail coefficients [5].

In matrix representation, low- and high-frequency filters are interconnected vectors of coefficients: a highfrequency filter is obtained from a low-frequency filter by writing its coefficients in reverse order and changing the sign of each even coefficient. Thus, if we denote the coefficients of the low-frequency filter as a vector $c$ of length $2 n$, then the wavelet transform of the signal will consist in multiplying the corresponding column vector by a matrix of the following form:

$$
\left(\begin{array}{cccccccccc}
c_{1} & c_{2} & c_{3} & \ldots & c_{2 n-1} & c_{2 n} & 0 & 0 & 0 & \ldots \\
-c_{2 n} & c_{2 n-1} & -c_{2 n-2} & \ldots & -c_{2} & c_{1} & 0 & 0 & 0 & \ldots \\
0 & 0 & c_{1} & c_{2} & c_{3} & \ldots & c_{2 n-1} & c_{2 n} & 0 & \ldots \\
0 & 0 & -c_{2 n} & c_{2 n-1} & -c_{2 n-2} & \ldots & -c_{2} & c_{1} & 0 & \ldots \\
\ldots & \ldots & \ldots & \ldots & \ldots & \ldots & \ldots & \ldots & \ldots & \ldots
\end{array}\right) \quad(7)
$$

To carry out the inverse transformation, it is necessary to multiply the vector of signal coefficients by the inverse matrix:

$$
\left(\begin{array}{ccccc}
c_{1} & -c_{2 n} & 0 & 0 & \ldots \\
c_{2} & c_{2 n-1} & 0 & 0 & \ldots \\
c_{3} & -c_{2 n-2} & c_{1} & -c_{2 n} & \ldots \\
\ldots & \ldots & c_{2} & c_{2 n-1} & \cdots \\
c_{2 n-1} & -c_{2} & c_{3} & -c_{2 n-2} & \cdots \\
c_{2 n} & c_{1} & \cdots & \ldots & \cdots \\
0 & 0 & c_{2 n-1} & -c_{2} & \cdots \\
0 & 0 & c_{2 n} & c_{1} & \ldots \\
0 & 0 & 0 & 0 & \ldots \\
\cdots & \ldots & \ldots & \ldots & \ldots
\end{array}\right)
$$

From the point of view of using a set of filters, discrete wavelet transform of a signal $x$ according to Malla's scheme are obtained by decomposing the signal through a low-frequency filter with an impulse response $g$ and a high-frequency filter $h$ as a result, the coefficients of approximation and detail are obtained, respectively (Fig. 1).

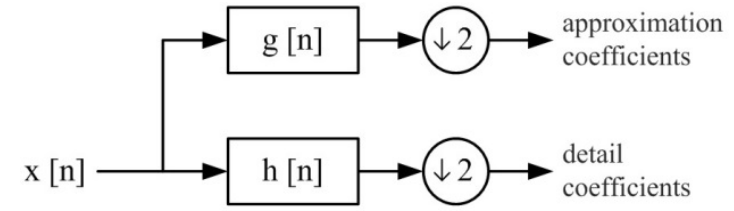

Fig. 1. Signal decomposition scheme for fast discrete wavelet transform (according to Malla’s scheme)

\section{HAAR AND DAUBECHIES WAVELETS}

The simplest and most widespread wavelets are the Haar and Daubechies wavelets.

Haar wavelets (HAAR wavelets) are orthogonal and well localized in space, but they are not smooth, symmetric in shape and do not have good localization in the frequency domain [6]. The basic wavelet function has the form of rectangular pulses and is set as follows:

$$
\psi(t)=\left\{\begin{array}{l}
1,0 \leq t<\frac{1}{2} \\
-1, \frac{1}{2} \leq t<1 \\
0, t \notin[0,1)
\end{array}\right.
$$

or in discrete representation:

$$
\psi_{m, k}=2^{m / 2} \psi\left(2^{m} t-k\right)
$$
[7].

where $m$ - scale parameter, and $k$ - shift parameter

The scaling function that determines the approximation of the signal is constant and described by the following expression:

$$
\varphi(t)=\left\{\begin{array}{l}
1,0 \leq t<1 \\
0, t \notin[0,1)
\end{array}\right.
$$

or discretely:

$$
\varphi_{j, k}=2^{j / 2} \varphi\left(2^{j} t-k\right)
$$

The Haar transform is a pair of filters that separate the signal into low-frequency and high-frequency components [8]. That is, each pair of adjacent elements of a one-dimensional signal is assigned two new signals: the approximating signal $a_{i}$, which is calculated as the half-sum of the elements, and the detail signal $b_{i}$ - half difference of adjacent elements. form:

The Haar transformation matrix has the following 


$$
H=\left(\begin{array}{cc}
\frac{1}{\sqrt{2}} & \frac{1}{\sqrt{2}} \\
\frac{1}{\sqrt{2}} & -\frac{1}{\sqrt{2}}
\end{array}\right)
$$

It has the property of orthogonality, so its inverse matrix can be obtained by transposition:

$$
H^{-1}=H^{T}=\left(\begin{array}{cc}
\frac{1}{\sqrt{2}} & \frac{1}{\sqrt{2}} \\
\frac{1}{\sqrt{2}} & -\frac{1}{\sqrt{2}}
\end{array}\right)^{T}=\left(\begin{array}{cc}
\frac{1}{\sqrt{2}} & \frac{1}{\sqrt{2}} \\
\frac{1}{\sqrt{2}} & -\frac{1}{\sqrt{2}}
\end{array}\right)
$$

The two-dimensional Haar transform in accordance with the Malla scheme is reduced to a composition of one-dimensional transformations: a one-dimensional wavelet transform is applied to each row and subsequently column of the two-dimensional matrix, as a result of which four matrices are obtained: approximating the original signal, as well as vertical, horizontal and diagonal detailing signals [3].

Since the Haar wavelet is not smooth, Ingrid Daubechies suggested using functions that are calculated iteratively. Such wavelets do not have an explicit analytical representation of a function by a single formula; they have orthogonality, compact support, and are asymmetric. The widespread use of these wavelets in digital signal processing has led to the possibility of using them to implement fast discrete wavelet transform.

One of the characteristics of Daubechies wavelets is the order $n$, which is defined as half the number of coefficients [9]. There are two variants of notation for Daubechies wavelets:

- $D N$, where $N$ - number of wavelet filter coefficients;

- $\quad d b n$, where $n$ - wavelet order.

With an increase in the order, the "smoothness" of the wavelets and the steepness of the cutoff of their frequency characteristics increase, therefore, the quality of signal decomposition and their reconstruction.

The coefficients of the Daubechies transformation matrix are found using the system of equations, which for the Daubechies wavelet $D N$ will contain $N$ equations: $\frac{N}{2}$ equations following from the orthonormality condition, $\frac{N}{2}$ and zero moment equations. For example, to find the values of the coefficients of the Daubechies transformation matrix $D 4$, the system of equations looks as follows:

$$
\left\{\begin{array}{c}
c_{1}^{2}+c_{2}^{2}+c_{3}^{2}+c_{4}^{2}=1 \\
c_{3} c_{1}+c_{4} c_{2}=0 \\
c_{4}-c_{3}+c_{2}-c_{1}=0 \\
c_{4}-2 c_{3}+3 c_{2}-4 c_{1}=0
\end{array}\right.
$$

To perform one-level two-dimensional wavelet decomposition with respect to a specific wavelet, the MATLAB Wavelet Toolbox provides the 'dwt2' command. The results of performing a two-dimensional wavelet transform in the MATLAB system are shown in Fig. 2.
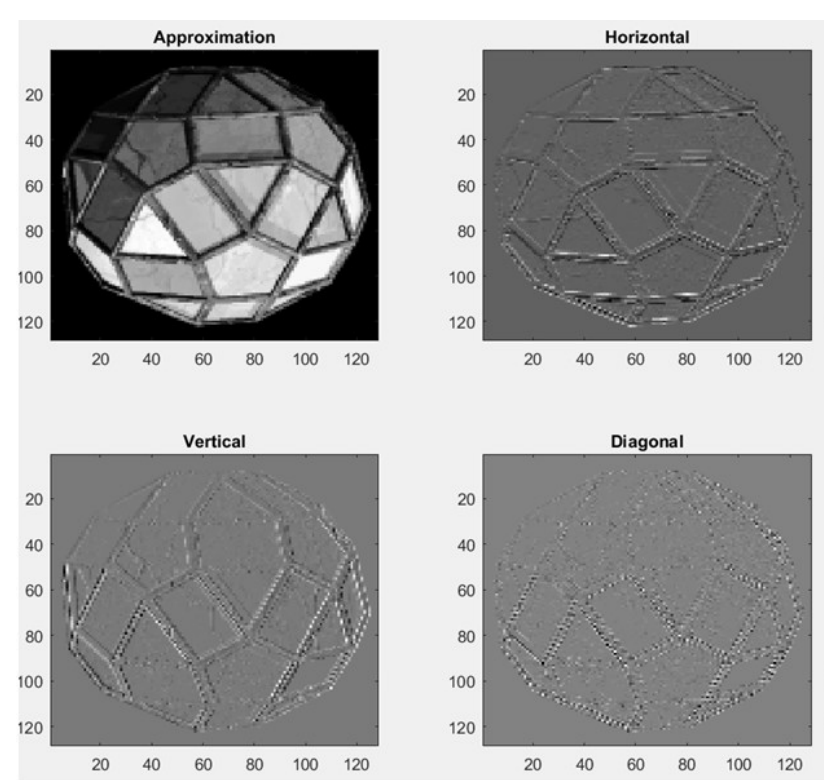

a)
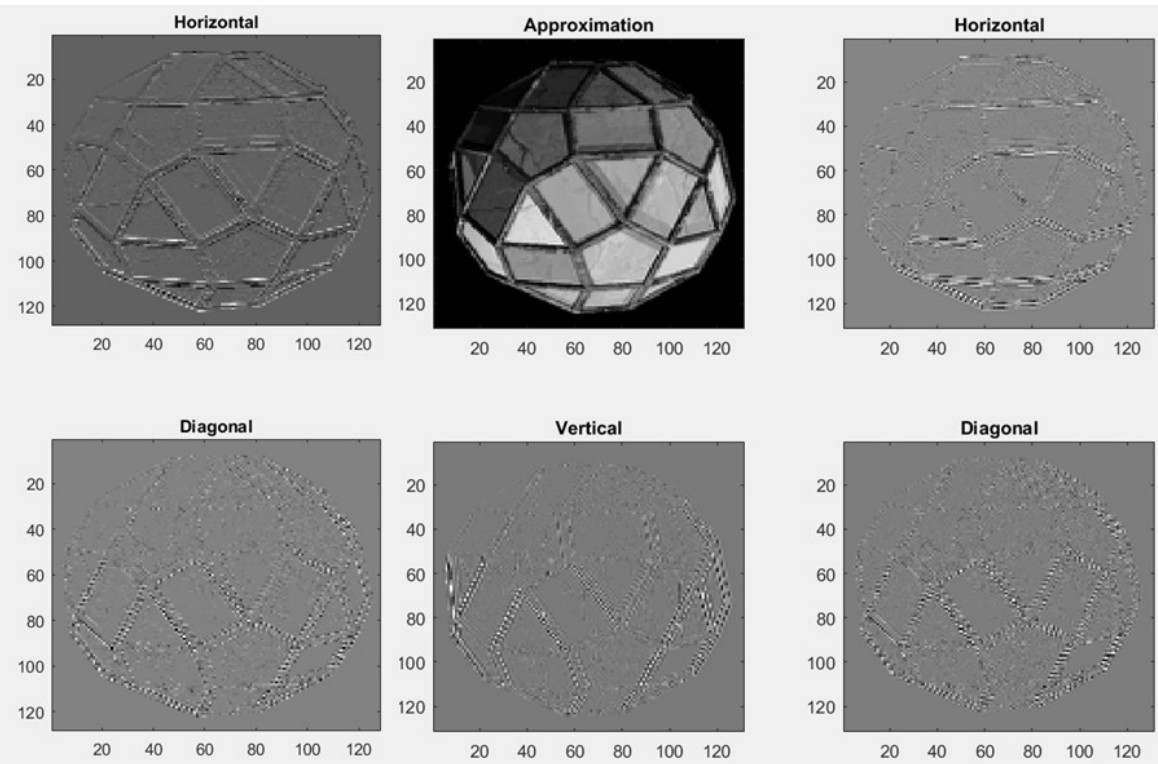

b)

Fig. 2. Two-dimensional transformation in the MATLAB system: a - with basic Haar wavelet, b - with basic Daubechies wavelet db4 
Environment. Technology. Resources. Rezekne, Latvia Proceedings of the $13^{\text {th }}$ International Scientific and Practical Conference. Volume 2, 174-180

\section{ApPlication of Methods of Progressive COEFFICIENTS’ VALUES (PCSM) IN INFORMATION COMPRESSION}

Wavelet transforms provide an efficient solution to the compression problem. However, in addition to this, the complete cascade sequence of two-dimensional signals' compression, or true compression, includes the iterative phases of quantization, encoding and decoding (Fig. 3) [10].

There are progressive coefficients' values (PCSM) techniques that combine wavelet decomposition and quantization. The main purpose of such methods is to transmit as soon as possible the most important piece of information about the signal, which gives the largest reduction in the discrepancy between the original and reconstructed signals [11]. Such methods are based on the following ideas:

- receiving an image with an increased resolution during decoding;

- obtaining a set of compression coefficients based on the length of the stored code;

- use in aggregate of wavelet decomposition to ensure sparsity and classical coding methods;
- using a tree structure for wavelet decomposition [12] - [14].

Some of the methods for progressive coefficients' values in MATLAB Wavelet Toolbox terminology are as follows:

- $\quad$ 'ezw' - the Shapiro method, or the Embedded Zerotree Wavelet method - combines stepwise thresholding and progressive quantization, focused on efficient coding in order to minimize the compression coefficient;

- 'spiht', 'spiht_3d' - the method of partitioning a set in hierarchical trees (Set Partitioning In Hierarchical Trees) - an improved version of the original EZW algorithm, which uses nested encoding, and also provides the best quality of a two-dimensional signal at any stage of decoding;

- 'stw' - Spatial-orientation Tree Wavelet method;

- ' ' $w d r$ ' - Wavelet Difference Reduction method;

- 'aswdr' - Adaptively Scanned Wavelet Difference Reduction method [10, 15].

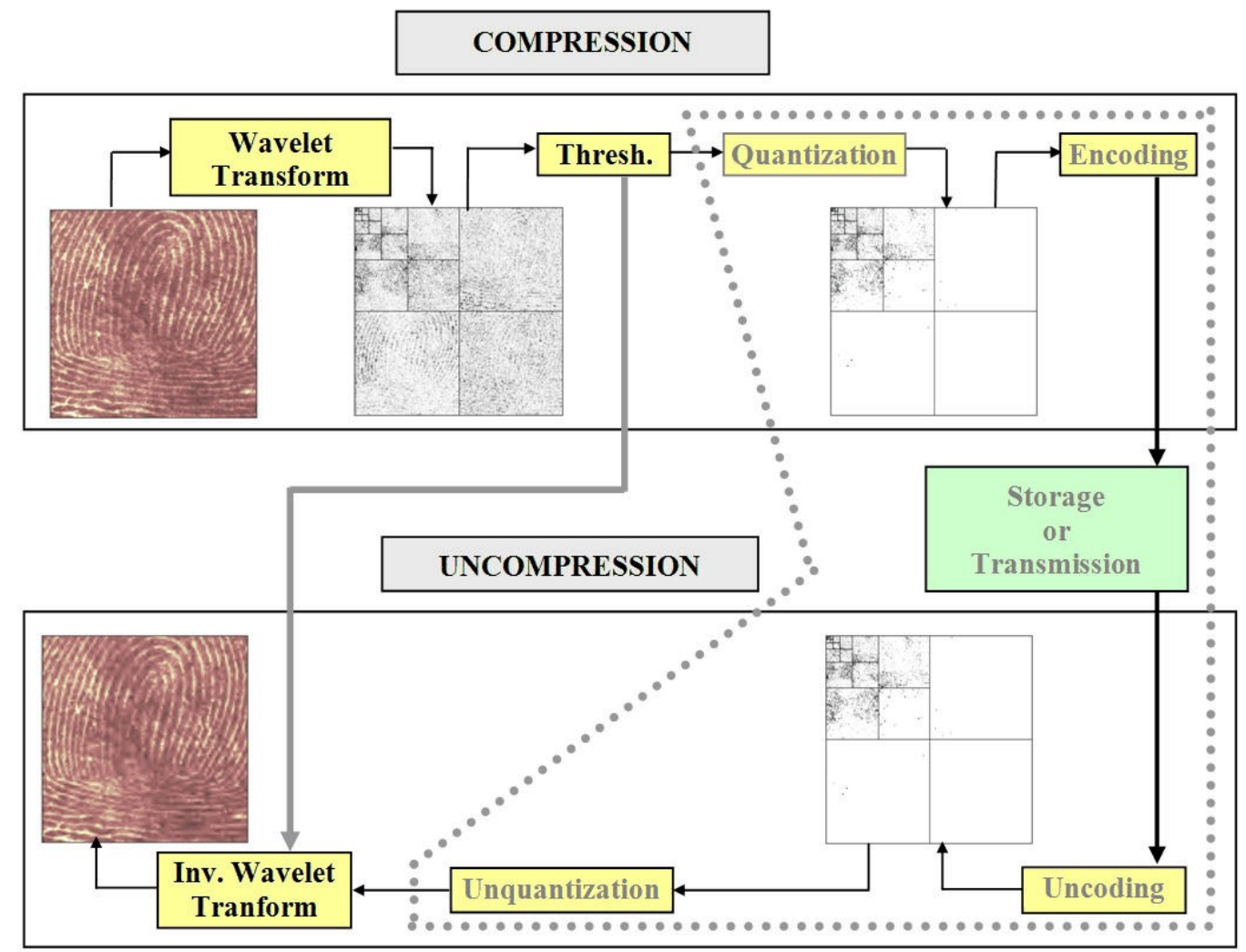

Fig. 3. Genuine compression scheme

The quality of data compression in the aggregate depends on the compression method, the selected wavelet and the number of iterations [16]. Fig. 4-7 show the compression results for the 'ezw' and 'spiht' methods using the Haar and Daubechies db4 wavelets in various combinations $[17,18]$. 

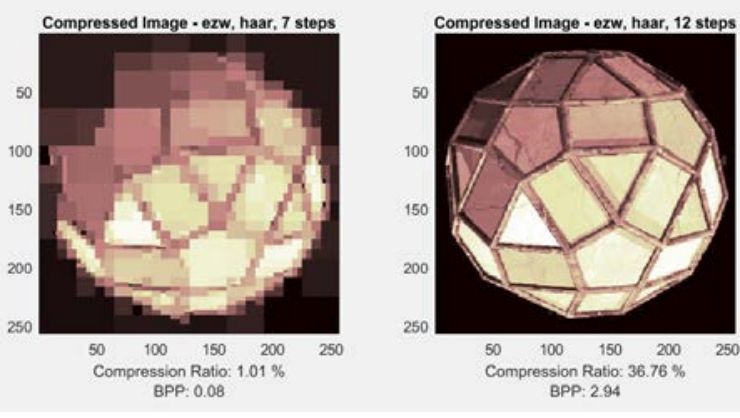

Fig. 4. Results of compression of a two-dimensional signal by the EZW method using the Haar wavelet
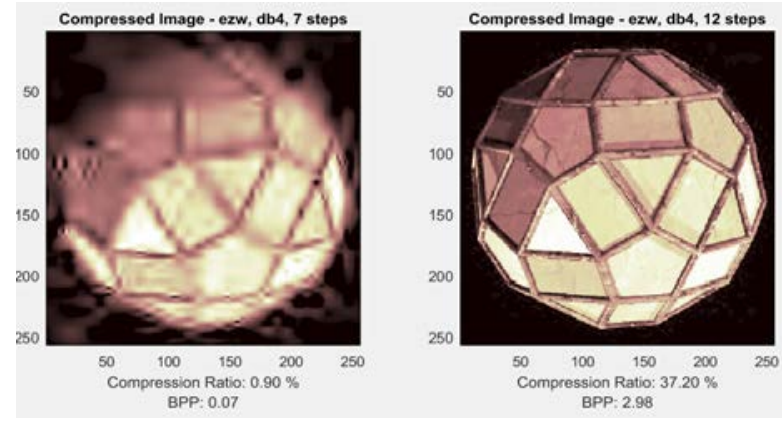

Fig. 5. Results of compression of a two-dimensional signal by the EZW method using the Daubechies wavelet db4
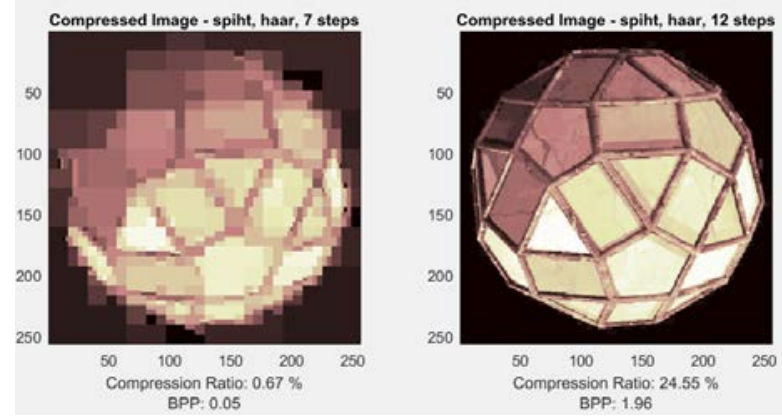

Fig. 6. Results of compression of a two-dimensional signal by the SPIHT method using the Haar wavele
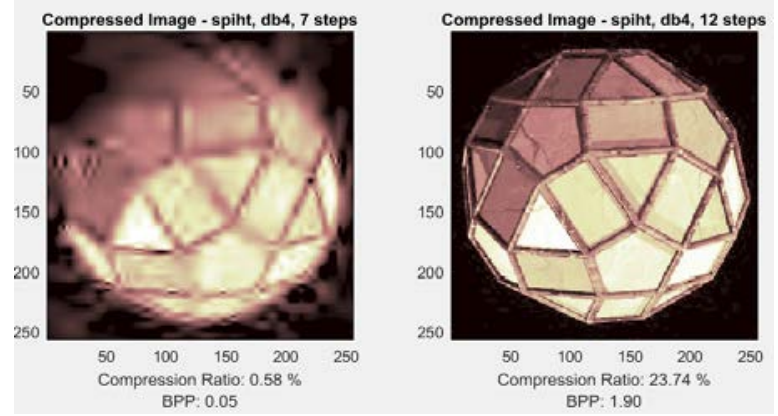

Fig. 7. Results of compression of a two-dimensional signal by the SPIHT method using the Daubechies wavelet db4

\section{CONCLUSION}

The use of wavelet transforms in data compression has a number of advantages: information redundancy is eliminated, data is restored with the same filters, and lossy compression is implemented simply by discarding unimportant details [19]. Although wavelet analysis is already the basis of some compression technologies, this direction of research remains relevant, because there is no single method for choosing a transformation algorithm and a basic wavelet [20, 21].

\section{REFERENCES}

[1] S. Verteshev, V. Konevtsov, "Processes control with fuzzy initial information in a complex of software design of digital control systems,” in Environment. Technology. Resources: Proceedings of the 11th International Scientific and Practical Conference on Engineering sciences and production technologies, Rezekne, 2017, vol. III, pp. 332-336.

[2] S. Verteshev, V. Konevtsov, "Direct digital control in a complex of software design of digital control systems,” in Environment. Technology. Resources: Proceedings of the 11th International Scientific and Practical Conference on Engineering sciences and production technologies, Rezekne, 2017, vol. III, pp. 337-342.

[3] Bauman National Library, "Wavelet transform,” 2016. [Online]. Available: https://ru.bmstu.wiki/Вейвлет-преобразование [Accessed: Feb. 27, 2021]. (in Russian)

[4] V. Konevtsov, I. Poletaev, S. Verteshev, "Discrete automatic schemes for ASC TP,” in Environment. Technology. Resources: Proceedings of the 10th International Scientific and Practical Conference on Engineering sciences and production technologies, Rezekne, 2015, vol. I, pp. 67-71.

[5] O. V. Nagornov et al., Wavelet analysis in examples. Moscow: NRNU MEPhI, 2010. (in Russian)

[6] Lossless data compression. Odessa: Odessa National Academy of Telecommunications named after A. S. Popov, 2006. (in Russian)

[7] V. P. Dyakonov, Wavelets. From theory to practice. Moscow: Solon-press, 2010. (in Russian)

[8] R. Gonzalez and R. Woods, Digital Image Processing. Moscow: Technosphere, 2012. (in Russian)

[9] T. E. Krenkel and M. A. Bazhanova, "Quantum Daubechies Wavelet Transform,” T-comm - Telecommunications and Transport, vol. 8, No. 12, pp. 35-40, 2014. (in Russian)

[10] N. K. Smolentsev, Fundamentals of Wavelet Theory. Wavelets in MATLAB. Moscow: DMK Press, 2005. (in Russian)

[11] N. M. Astafieva, "Wavelet analysis: the foundations of the theory and examples of application," Advances in Physiological Sciences, vol. 166, No. 11, pp. 1145-1170, 1996. (in Russian)

[12] D. Andreev, S. Lyokhin, L. Motaylenko, and S. Verteshev, "Models and algorithms for constructing a formalized description of production technologies," in Environment. Technology. Resources: Proceedings of the 12th International Scientific and Practical Conference on Information Technologies, Rezekne, 2019, vol. II, pp. 21-27.

[13] I. Antonov, I. Bruttan, D. Andreev, and L. Motaylenko, "The method of automated building of domain ontology," in Environment. Technology. Resources: Proceedings of the 12th International Scientific and Practical Conference on Information Technologies, Rezekne, 2019, vol. II, pp. 34-37.

[14] D. Andreev, S. Lyokhin, V. Nikolaev, and O. Poletaeva, "Development of software for design ontological representations of production technologies," in Environment. Technology. Resources: Proceedings of the 12th International Scientific and Practical Conference on Information Technologies, Rezekne, 2019, vol. II, pp. 28-33.

[15] D. Salomon, Data, Image and Sound Compression. Moscow: Technosphere, 2004. (in Russian)

[16] Yu. Taranenko, "Wavelet analysis. Part 1,” 2019. [Online]. Available: https://habr.com/ru/post/451278/ [Accessed: Feb. 27, 2021]. (in Russian)

[17] I. Daubechies, Ten Lectures on Wavelets. Izhevsk: Research Center "Regular and Chaotic Dynamics", 2001. (in Russian) 
Environment. Technology. Resources. Rezekne, Latvia Proceedings of the $13^{\text {th }}$ International Scientific and Practical Conference. Volume 2, 174-180

[18] Wikipedia - the free encyclopedia, "Haar Wavelet," 2020. [Online]. Available: https://ru.wikipedia.org/wiki/ Вейвлет_Хaаpa [Accessed: Feb. 27, 2021]. (in Russian)

[19] I. M. Dremin, O. V. Ivanov, and V. A. Nechitailo, "Wavelets and their use,” Advances in Physiological Sciences, vol. 171, No. 5. pp. 465-501, 2001. (in Russian)
[20] V. I. Vorobiev and V. G. Gribunin, Theory and Practice of Wavelet Transform. St. Petersburg: VUS, 1999. (in Russian)

[21] Bauman National Library, "Daubechies wavelets," 2016. [Online]. Available: https://ru.bmstu.wiki/Вейвлеты_Добеши [Accessed: Feb. 27, 2021]. (in Russian) 